(C) 2016 IEEE. Personal use of this material is permitted. Permission from IEEE must be obtained for all other uses, in any current or future media, including reprinting/republishing this material for advertising or promotional purposes, creating new collective works, for resale or redistribution to servers or lists, or reuse of any copyrighted component of this work in other works.

\title{
An Automated Test Equipment for Characterization of emerging MRAM and RRAM arrays
}

\author{
Alessandro Grossi, Cristian Zambelli, Member, IEEE, Piero Olivo, Paolo Pellati, Michele Ramponi, \\ Christian Wenger, Jérémy Alvarez-Hérault and Ken Mackay
}

\begin{abstract}
In this paper it is presented a test equipment for the characterization of two different emerging memory technologies like the Thermally Assisted Switching-Magnetic Random Access Memory (TAS-MRAM) and the Resistive Random Access Memory (RRAM). The instrument is developed to allow a fast characterization of test array structures and can be potentially adapted for any other non-volatile memory generation. The hardware architecture is based on a PCI S5933 chipset being the local bus interface of a x86-PC that communicates with the units of the system like $14 \mathrm{bits} / 100 \mathrm{MHz}$ arbitrary waveform generators and 12 bits/70 $\mathrm{MHz}$ programmable measurement units. A user-friendly software interface developed in LabVIEW has been implemented to allow large flexibility in changing the test parameters and a fast analysis of the test results. The instrument performance has been evaluated performing the typical non-volatile memory tests such as endurance and disturbs characterizations, running test flows up to $\mathbf{3 2 0}$ hours for MRAM devices and up to 6137 hours for RRAM devices.
\end{abstract}

Index Terms-Automated test equipment, non-volatile memory, reliability, thermally assisted switching, MRAM, RRAM

\section{INTRODUCTION}

The development and the testing of non-volatile memories (NVM) requires extensive characterizations that only a dedicated Automated Test Equipment (ATE) is able to handle. Different features might be required depending on the maturity level of the technology being characterized. Indeed, for a well-established NVM like the Flash technology, where the integration density is the highest, the most desired capability of a test environment is to run standard test algorithms in a highly parallel yet efficient way [1]. On the contrary, for an emerging technology where the physical mechanisms ruling the storage have to be explored, and the investigations are carried on test array structures that are commonly integrated without any internal circuitry (e.g., I/O interfaces, charge pumps, sense amplifiers, etc.) except what is used for cell addressing, a maximum flexibility is sought in terms of signals and timings features applied on the NVM [2], [3].

This work was supported in part by the European Community, FP7 Programme, through the ATHENIS-3D project under Grant 619246 and by the European Union's H2020 research and innovation programme under grant agreement $\mathrm{N}^{\circ} 640073$

A. Grossi, C. Zambelli and P. Olivo are with Dipartimento di Ingegneria, Università degli Studi di Ferrara, Via Saragat 1, Ferrara (Italy), 44122 (e-mail: alessandro.grossi@unife.it).

P. Pellati and M. Ramponi are with Active Technologies, Via Bela Bartok 29/B, Ferrara (Italy), 44124

Ch. Wenger is with IHP, Im Technologiepark 25, 15236 Frankfurt (Oder), Germany

J. Alvarez-Hérault and K. Mackay are with Crocus Technology Grenoble, 4 place Robert Schuman, 38025 Grenoble Cedex, France.
The testers for NVM traditionally employed DC instruments, such as source measurement units, after the use of pulse generators to program/erase the memory cells. This approach required some type of switch to alternatively apply the DC or the pulse signal to the memory under test. Occasionally, oscilloscopes were used to monitor the goodness of the signal applied to the memory, but this approach became unfrequent due to the different setup required for the DC measurements. Moreover, that technique limited the possibility to monitor currents and voltage at the same time, requiring custom complex measurement systems that were often cumbersome and introduced time-consuming data extraction procedures. Also, obtaining system-level calibration across multiple systems was impossible [4]. The present instrumentation for NVM testing allows gaining a better understanding of the memory functionalities by improving the characterization capabilities, but this comes with a trade-off. When large test populations (i.e., memory arrays) are tested then only digital-based pattern testing is possible, otherwise analog current/voltage testing is possible only on a small amount of memory cells.

In this paper we present an ATE dedicated to the characterization of two emerging non-volatile memory technologies like 1 kbits TAS-MRAM and 4 kbits RRAM arrays. The proposed test equipment takes into account the specific requirements of both technologies, while aiming at achieving the performance of a production-line tester, guaranteeing fast and accurate testing on large ensembles of memory cells. The proposed ATE allows performing the typical tests that are demonstrated by commercial platforms specifically tailored for MRAM and RRAM technologies characterization such as the one in [5] [8]: endurance testing (performed in up to 320 hours for 1 kbits TAS-MRAM and up to 6137 hours for 4 kbits RRAM memories), hysteresis (R-V curves in TAS-MRAM performed in one hour), I-V curves in RRAM performed in less than one hour, read disturb (one million disturb cycles in less than 1200 hours). Additional tests like breakdown voltage, switching voltages with respect to pulse widths, switching probability distribution with respect to applied voltage, low/high state resistance distribution and error rate testing can be performed as well. These characterizations are achieved by the proposed ATE without sacrificing the accuracy and ease of operation of the system, but limiting the possibility to measure transient $\mathrm{read} / \mathrm{write}$ times for specific operations. This shortcoming of the system can be addressed in a future development of the ATE for other NVMs where the transient characterization is critical.

The instrument architecture has been developed with a 


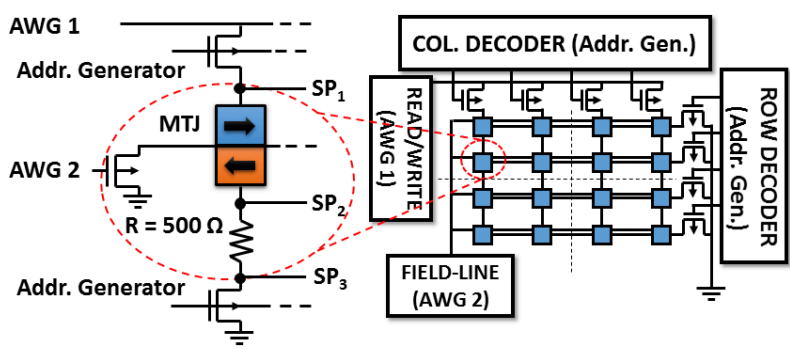

Fig. 1. TAS-MRAM memory cell (left) and array architecture (right) exploited in this paper.



Fig. 2. RRAM memory cell (left) and array architecture (right) exploited in this paper.

twofold purpose: $i$ ) provide an environment for highly paralle testing in order to speed-up the long-term reliability characterization through the usage of high-speed voltage waveform generators (14 bits/100 MHz) and parallel measurement units (12 bits/70 MHz) managed by a 32 bits RISC processor; ii) provide all the flexibility required in terms of operation modes that are requested by the exploration of an emerging technology. A software interface has been implemented using LabVIEW tightly coupled with a low-level driver. This solution allows implementing specific features to statistically identify failures and abnormal technology behaviors.

The advantages of the proposed ATE with respect to the state-of-the-art testers [5], [9]-[11] are modularity, flexibility and portability. The ATE can be easily modified adding/replacing waveform generators to satisfy specific requests for the memory technology without requiring any successive calibrations, granting de-facto its applicability to other NVM technologies. The performance and effectiveness of the proposed ATE has been preliminary demonstrated in [12], [13] and here is fully validated on different MRAM and RRAM test arrays.

\section{ATE REQUIREMENTS}

The paved capabilities of the TAS-MRAM in terms of performance and reliability pose however several challenges in the development of an ATE suitable for their characterization. The first issue is related to the current supply required by the cells in order to reliably switch between different logical states [14]. Each memory cell needs two current sources: one driving the heating phase (i.e., thermal assistance), and one driving the effective magnetic field switch. Their current value strictly depend on the parallelism to be achieved. Therefore, waveform generators with a high current output stage and a high slew rate are required to meet the fast operation feature of the TAS-MRAM technology, that is in the range of $200 \mathrm{~ns}$ $\div 20 \mu s$.

The second issue is due to the extreme long-term reliability offered by the technology [15]-[17]. TAS-MRAM cells exhibit an endurance capability up to several millions switching cycles (i.e., orders of magnitude higher than a traditional flash technology), therefore to monitor potential reliability threats coming either from the manufacturing process or from the chosen write algorithm it is required to repeatedly apply sequences of read/write operations with a perfect synchronization of the tasks to perform during the test. Moreover, it must be guaranteed a non-blocking time of the operations that extract the characterization data from the memory (i.e., read operation) both to avoid testing limitations and to allow the user to interact with the ATE during test (e.g., to extract statistical data).

The capabilities of RRAM memory cells in terms of current supply and fast operation features are not as strict as TASMRAM, therefore an ATE with flexibility and performance able to characterize MRAM technology is also suitable for testing RRAM arrays.

\section{TEST CHIP SPECIFICATIONS}

Among the emerging technologies that are under consideration for a possible replacement of the standard Flash [18] memories in embedded environments, the Thermally Assisted Switching - Magnetic Random Access Memory (TAS-MRAM) [19]-[21] and Resistive Random Access Memory (RRAM) [22]-[24] represent two good candidates. In the former technology the information storage mechanism is based on the magnetization switch of a magnetic material controlled by applying a current, that generates a magnetic field either parallel or antiparallel to a fixed reference layer thus showing a defined resistance [25], [26]. In the latter technology the conductance of a Metal-Insulator-Metal (MIM) stack can be electrically modified creating or disrupting a conductive filament within the stack through Set and Reset operations, respectively. The presence or absence of such filament translates into a resistance variation of the MIM cell that defines the memory cell state. Both technologies are already in a phase that calls for their evaluation at an integrated array level.

\section{A. MRAM}

The MRAM test chips tested by the designed ATE are 1 kbits TAS-MRAM arrays where only cells and decoders are present, organized in 4 banks with 8 wordlines and 32 bitlines each. The cells' architecture is a 1T-1J, integrated into a CMOS process from Crocus Technology, that is selected via a 10-bit multiplexer (see Fig. 1) [27]. To prove the capabilities of the proposed ATE to handle different TASMRAM technologies two different generations of the arrays have been characterized, hereafter indicated as Sample A and Sample B. The main difference in the two technologies is the stack of materials integrated in the cell structure, leading to different hysteresis characteristics in the magnetic materials 
and read/write speed of the array. The specifications of both test array architectures are the following:

- each memory cell in the array requires two signals during a write operation applied on dedicated pads. The first signal (AWG 1 in Fig. 1) drives the heating phase of the write procedure and ranges from $0 \mathrm{~V}$ up to $2 \mathrm{~V}$, whereas the second signal (AWG 2 in Fig. 1) drives the effective magnetization switch by applying either a positive or a negative voltage ranging from $0 \mathrm{~V}$ up to $\pm 5 \mathrm{~V}$.

- During the read operation, after applying a fixed voltage ranging from $0 \mathrm{~V}$ up to $0.5 \mathrm{~V}$ on $\mathrm{AWG} 1$, three different voltages must be sensed on dedicated measurement pads that represent three sensing positions on the memory cell: the voltage on top of the magnetic element $\left(\mathrm{SP}_{1}\right)$, the voltage below it $\left(\mathrm{SP}_{2}\right)$, and voltage between the select transistor and a fixed $500 \Omega$ resistor $\left(\mathrm{SP}_{3}\right)$. The combination of the voltages allows extracting the resistance value of the memory cell and hence its logical state.

The timings are usually $200 \mathrm{~ns} \div 20 \mu$ s for the writing operations and $20 \mu \mathrm{s}$ for the read operation. For the former it is important to guarantee that the signal driving the switching is synchronized with the heating voltage and that it ends 100 ns $\div 10 \mu$ s after the end of the heating signal.

\section{B. RRAM}

The RRAM test chips are 4 kbits arrays composed by a memory cells matrix, a bitline/sourceline (BL/SL) and wordline (WL) decoder and an operation control circuitry (see Fig. 2) [6]. The 1T-1R memory cells are constituted by a select NMOS transistor manufactured with a $0.25 \mu \mathrm{m}$ BiCMOS technology whose drain is in series to the MIM stack. The variable MIM resistor is composed by $150 \mathrm{~nm}$ TiN top and bottom electrode layers, a $7 \mathrm{~nm}$ Ti layer, and a $8 \mathrm{~nm} \mathrm{HfO}_{2}$ layer. To activate such a switching behavior, some technologies require a preliminary Forming operation in order to create the conductive path for the first time [28], [29]. The specifications of such architecture are the following:

- Forming, Set and Reset operations require the application of two signals on dedicated pads. The first signal (AWG 1 ) is applied on the WL of the selected $1 \mathrm{~T}-1 \mathrm{R}$ cell to turn on the NMOS transistor and to define the current compliance, whereas the second signal (AWG 2) is applied



Fig. 4. Control panel of the RISC $\mu \mathrm{P}$ and of the digital sequencer. In this plot the AWG and PMU triggers are set for a read operation by the sequencer.

either on BL (during Forming and Set) or SL (during Reset) of the selected cell in order to create or break the conductive filament, respectively. The WL signal ranges from $1.5 \mathrm{~V}$ up to $2.8 \mathrm{~V}$, whereas BL and SL signals range from $0.2 \mathrm{~V}$ up to $3.5 \mathrm{~V}$.

- In read operation, while applying a fixed voltage pulse of $1.5 \mathrm{~V}$ on WL (AWG 1) and $0.2 \mathrm{~V}$ on BL (AWG 2), the current flowing through the BL must be sensed in order to extract the resistance value of the memory cell and hence its logical state.

The operations can be performed by single pulses or sequence of pulses with lengths between $1 \mu \mathrm{s} \div 100 \mu$ s for Forming, Set and Reset and $10 \mu \mathrm{s}$ for the read operation [30]. It is important to guarantee the synchronization between WL and BL/SL signals in all operations in order to correctly switch the devices. Moreover, during Read operation the voltage sensing must be correctly synchronized with WL and BL pulses.

\section{ATE HARDWARE ARCHITECTURE}

The instrument architecture is depicted in Fig. 3. The ATE is composed by: a PCI controller, a RISC $\mu P$ with a sequencer,

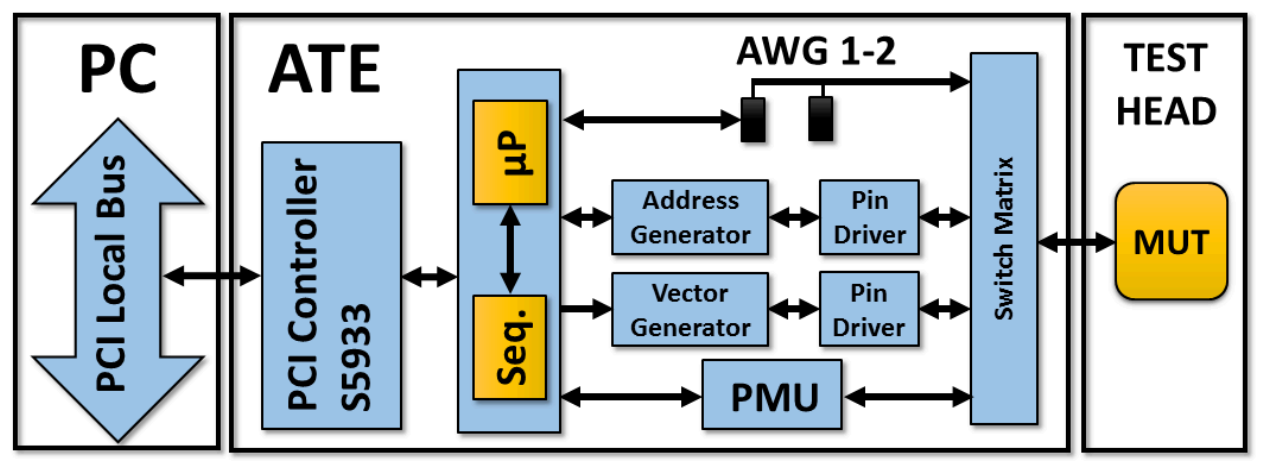

Fig. 3. ATE architecture block diagram. 
pin drivers for the power supply and the address/vector generator unit, two Arbitrary Waveform Generators (AWGs), and a multi-channel Programmable Measurement Unit (PMU).

\section{A. PCI Controller}

The ATE is controlled by a standard x86-PC through the PCI local bus installed on the motherboard. The S5933 PCI controller and the control logic of the digital instrument section are mounted on a board to be plugged into the computer. The choice of the PCI local bus as an interface for the ATE allows considering the Memory Under Test (MUT) as an extension of the x86-PC memory, thus allowing direct addressing and reading.

\section{B. Pin drivers}

To correctly provide the power supply to the MUT connected to the ATE, and to provide the voltage to the address and the vector generator units (i.e., the units responsible for creating the signals that select the cells in the arrays) a pin drivers unit has been implemented. This component provides the matching between the PCI controller and the MUT bias requirements.

\section{RISC $\mu P$ and sequencer}

The RISC processor mounted inside the ATE allows sharing the tasks with the $\mathrm{x} 86-\mathrm{PC}$ in order to optimize the system resources without loosing programming flexibility. The ATE core is implemented on a high performance FPGA. The digital sequencer unit coupled with the processor drives the digital control signals of the peripherals connected to the ATE (i.e., it provides the trigger for the AWGs and the PMU). The entire unit works at $400 \mathrm{MHz}$ allowing a $2.5 \mathrm{~ns}$ time resolution. Fig. 4 shows $\mu \mathrm{P}$ and sequencer control panel, showing the control signals applied during a read operation to trigger the voltage waveform generation by the AWGs with the timings required by the test chip specifications immediately followed by the PMU measurement.

\section{D. $A W G$}

The AWG units are the hardware components that drive the signals used during both write and read operations. Their design must fulfill all the tight requirements of the two technologies considered in this work, namely the high current output and the precision in generating short voltage pulses.

The block diagram of an AWG unit and its realization is shown in Fig. 5. The 14-bits digital samples of the voltage waveform to be generated, that can be graphically edited by the user with the ATE software interface, are stored in a highspeed static RAM. During measurement, on a trigger signal edge generated by the digital sequencer, the memory content is read in sequence and waveform samples are converted by a high-speed digital-to-analog converter (DAC) that operates at $100 \mathrm{MHz}$. A high-speed current feedback output buffer featuring a $1000 \mathrm{~V} / \mu$ s slew rate converts the DAC output current to voltage. The maximum current that can be drawn by an AWG unit is up to $500 \mathrm{~mA}$. The AWG clock speed defines a)

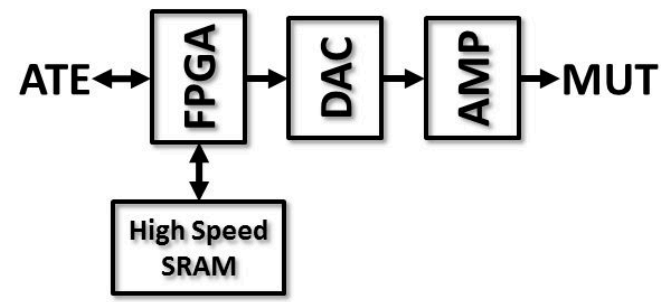

b)



Fig. 5. Arbitrary waveform generator block diagram (a) and picture (b).

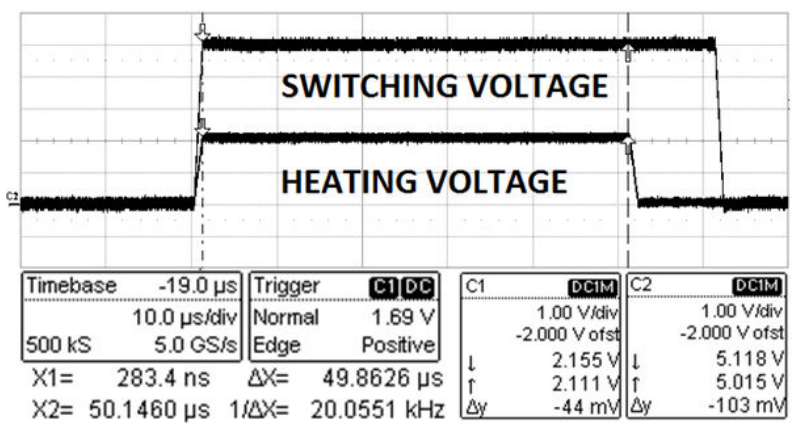

Fig. 6. Measured voltage waveforms generated by the AWG units during a write operation.

the ATE limitations in waveform generation, consisting of a minimum pulse voltage length of $10 \mathrm{~ns}$ featuring a minimum rise/fall time of $9 \mathrm{~ns}$. Its overall resolution is down to $1 \mathrm{mV}$. An example of voltage waveforms generated during the write operation on a TAS-MRAM array is shown in Fig. 6.

\section{E. Multi-channel PMU}

The PMU unit is used to sense the voltage on the TASMRAM sense pads and RRAM BL pin. The architecture of a single PMU channel is depicted in Fig. 7. Eight parallel independent channels are integrated in the ATE, each one featuring a signal conditioning amplifier with a high signal-tonoise ratio (AMP), a fast voltage measurement unit, a 12-bits ADC triggered directly by the digital sequencer, and a postprocessing buffer unit to temporary store the measurement data to be evaluated by the ATE software. The operating frequency of the PMU is $70 \mathrm{MHz}$ (14.3 ns resolution time) featuring a current measurement error less than $1 \%$. The ATE features a minimum read time of $40 \mathrm{~ns}$, limited by both AWG triggering and PMU clock speed. The gain and the offset adjustments of the PMU are stored in an EEPROM memory that is read at system start-up. 


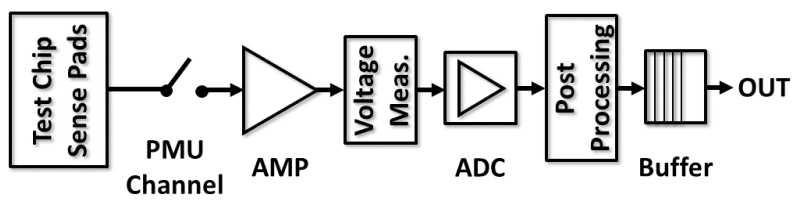

Fig. 7. Block diagram of a single PMU channel.

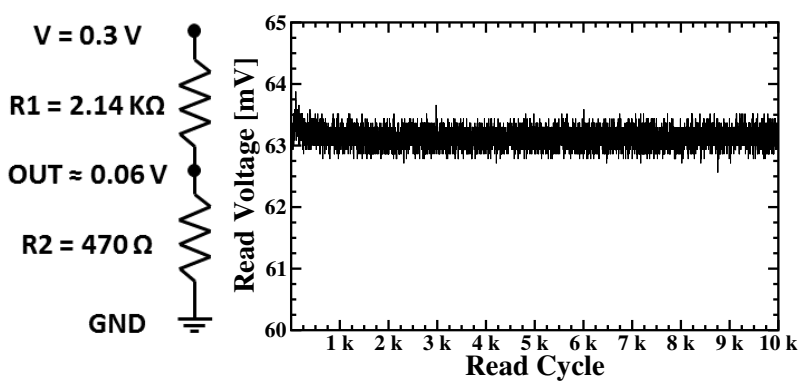

Fig. 8. Resistance reading: test setup (left) and sensing pad resistance reading (right). a)

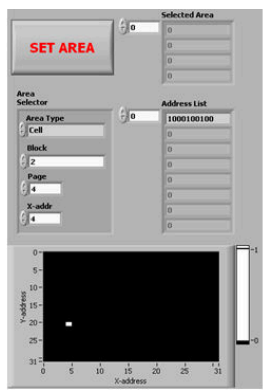

b)

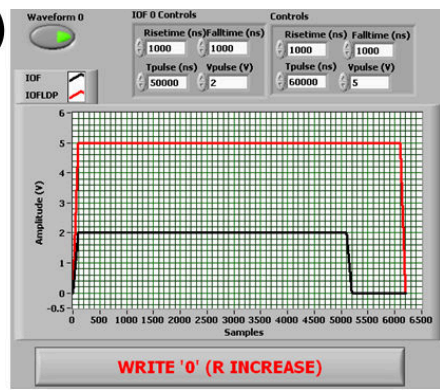

Fig. 9. GUI panel of the ATE for test population selection (a) and AWG setup for the write operations (b).

10000 consecutive read operations on three out of eight PMU channels have been performed using a test setup that mimic the resistance configuration of a TAS-MRAM memory cell. A voltage divider constituted by the resistances $\mathrm{R} 1=$ $2.14 k \Omega$ and $\mathrm{R} 2=470 \Omega$ (see Fig. 8a) is connected to the sense pads. The results obtained show an average displacement from the theoretical value equal to $3 \mathrm{mV}$, and a measurement uncertainty of $137 \mu \mathrm{V}$ ) as shown in Fig. 8 (b).

\section{ATE SOFTWARE INTERFACE}

The software interface is hierarchically organized in two different interacting layers: the low-level routines that directly communicate with the ATE hardware components, and the high-level libraries that interacts with the user through a Graphical User Interface (GUI). The low-level routines are written in $\mathrm{C}$ language to ensure efficiency both in terms of speed and memory requirements, whereas the high-level libraries have been implemented by using the National Instruments LabVIEW tool. The user can control any instrument operation and modify the ATE hardware parameters accordingly. It is possible to vary the write operation waveforms, the speed of the read operation, and many other parameters by simply acting on the GUI panels provided within the software interface (see an example in Fig. 9). a)

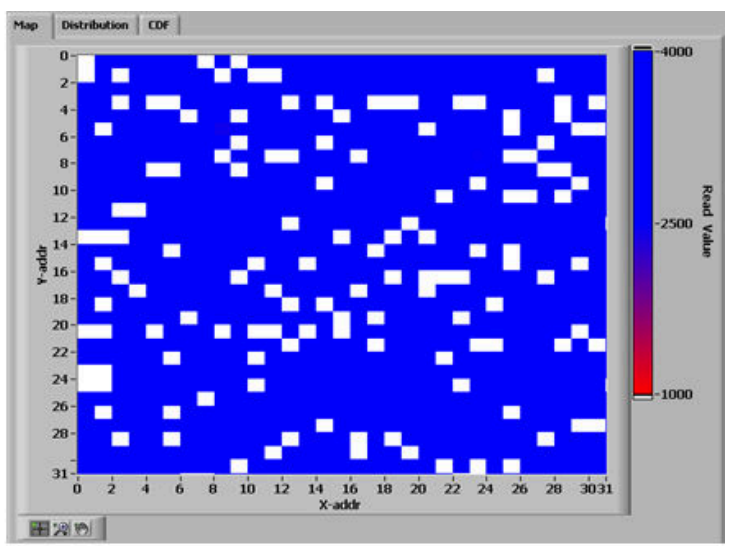

b)

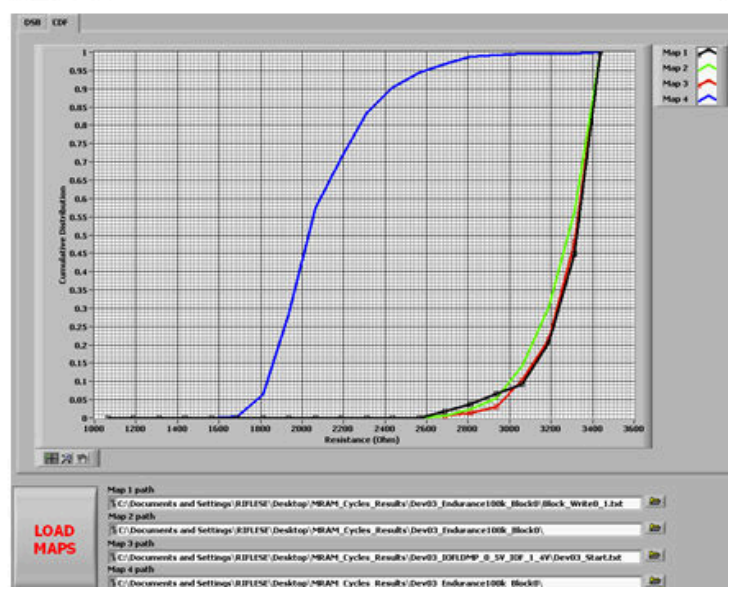

Fig. 10. Resistance map measured in a full TAS-MRAM chip reading operation on the user interface (a) and post-analysis tool used for statistical analysis (b).

The GUI also allows selecting the test population within the memory array by implementing an address scrambler that may choose from single cells, array wordlines, banks and full chip. By interacting with the low-level routines the ATE software identifies the degree of parallelization requested by the user and sets the hardware resources accordingly. Moreover, an environment for graphically visualizing the test sequence is provided through LabVIEW, and a post-analysis tool for immediate data characterization after test. An example is given in Fig. 10 where the tool is used to locate the cells of an entire TAS-MRAM array that failed to switch due to a breakdown condition under very high voltage stress. The data are also available after the test execution in different formats readable by the most common data processing tools.

\section{EXPERIMENTAL RESULTS AND ATE PERFORMANCE}

The ATE functionality and performance have been characterized through a set of tests applied on different TAS-MRAM and RRAM arrays. In order to show the ATE flexibility, the results obtained both on single cell subsets and full chip characterizations are reported. Typical NVM tests suitable for evaluating memory cells intrinsic reliability were performed on both technologies, such as:

- Hystheresis analysis (TAS-MRAM) and I-V curves (RRAM): they are obtained by measuring the read resistance while varying the voltage applied on the AWG 


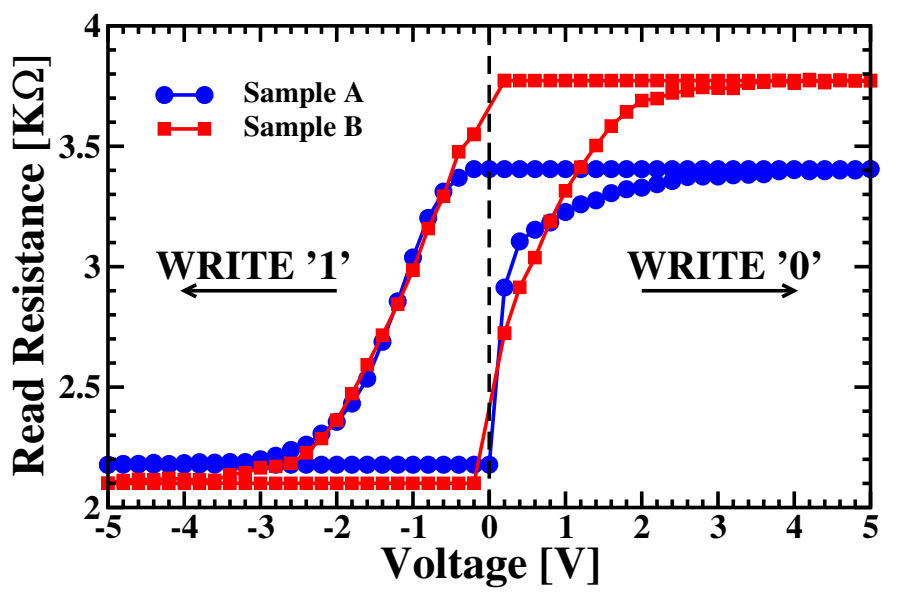

Fig. 11. Hysteresis characteristics of a single cell in Sample A and Sample B TAS-MRAM array.

connected to the field line drivers in TAS-MRAM and to the BL/SL in RRAM. These tests are useful to understand possible asymmetries in the writing process in TASMRAM and the Set/Reset switching dynamics in RRAM, while providing an estimate of the technology write speed.

- Read disturb test: it consists in consecutive read operations applied on the same set of cells in the array. This test is also useful in understanding whether or not the read operation could be a blocking factor for the ATE performance since the read operation cannot be parallelized.

- Endurance test: it consists in repeated write operations interleaved by a readout operation at fixed cycles. This test combines the evaluation of the ATE resources parallelization capabilities and the read operation constraints.

\section{A. MRAM}

Fig. 11 shows the hysteresis characteristic of a single TASMRAM cell in both array technologies. A complete analysis performed on one cell in the array by increasing the voltage from $0 \mathrm{~V}$ up to $5 \mathrm{~V}$ in $0.2 \mathrm{~V}$ steps for both write operations can be performed in less than 5 seconds per cell for both TAS-MRAM technologies.

A test of $1 \mathrm{M}$ disturb cycles applied on full arrays requires up to 440 hours for Sample A arrays and 77 hours for Sample $\mathrm{B}$ arrays which are a reasonable time considering that similar tests performed on single cell structures take almost the same time [17]. The results of the test are shown in Fig. 12.

Finally, to further test the efficiency of the tester, a $1 \mathrm{M}$ endurance switching cycling has been performed on a full chip. The test lasted 320 hours for Sample A technology and 56 hours for Sample B technology proving once again the goodness of the proposed measurement system. The results of the endurance characterization are shown in Fig. 13, where the average read resistance and standard deviation for both logical states are shown. The cumulative resistance distributions of Sample A (left) and sample B (right) TAS-MRAM array blocks in the different logical states at fixed switching cycles



Fig. 12. Average read resistance and standard deviations for both logical states measured during a $1 \mathrm{M}$ cycles read disturb test on a full Sample A TAS-MRAM array. Similar results are obtained for Sample B arrays.
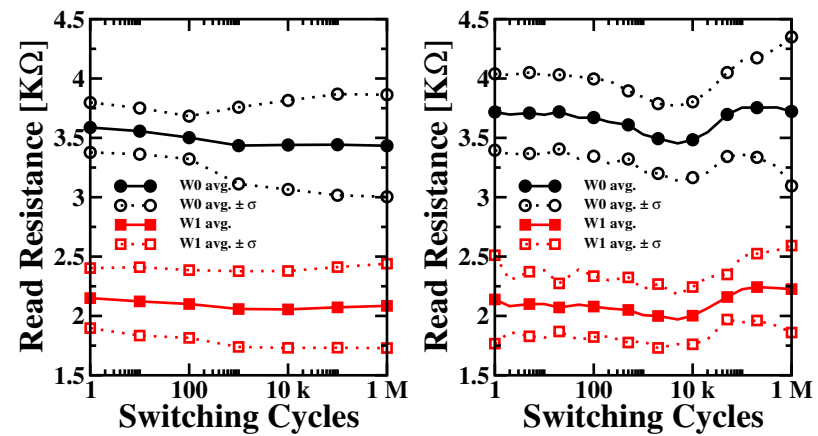

Fig. 13. Average read resistance and standard deviations of Sample A (left) and sample B (right) TAS-MRAM array blocks at fixed switching cycles measured during an endurance test constituted by $1 \mathrm{M}$ switching write cycles.
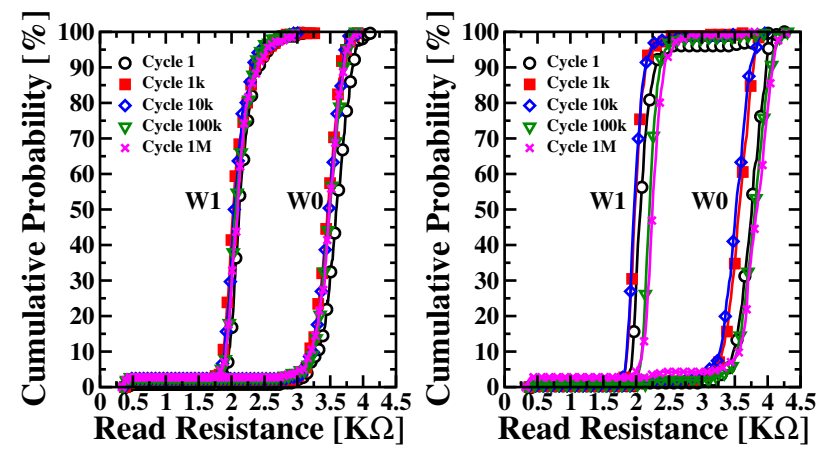

Fig. 14. Cumulative resistance distributions of Sample A (left) and sample B (right) TAS-MRAM array blocks in the different logical states at fixed switching cycles measured during an endurance test constituted by $1 \mathrm{M}$ switching write cycles.

measured during an endurance test are reported in Fig. 14. It is worth to point out that the tester accuracy is not impacted by the TAS-MRAM read/write speed, since there are no measurement artifacts introduced by the ATE in the statistical analysis of the arrays. A resume of the performance feature of the proposed ATE is presented in Table I. 
TABLE I

MEASURED ATE PERFORMANCE EVALUATED ON TAS-MRAM FULL CHIP TESTS

\begin{tabular}{|l|c|c|}
\hline Test & $\begin{array}{c}\text { Time [h] } \\
\text { Sample A }\end{array}$ & $\begin{array}{c}\text { Time [h] } \\
\text { Sample B }\end{array}$ \\
\hline Hysteresis analysis & 1.27 & 1.20 \\
Read disturb (1e6 cycles) & 440 & 77 \\
Endurance (1e6 cycles) & 320 & 56 \\
\hline
\end{tabular}

\section{B. RRAM}

In order to observe the RRAM cells switching dynamics, Set and Reset I/V characteristics have been measured with a DC sweep on 30 memory cells in the array, increasing the BL/SL voltage from 0 to $3.5 \mathrm{~V}$ with step voltage of $0.1 \mathrm{~V}$, and a WL voltage of $1.5 \mathrm{~V}$ in Set $2.5 \mathrm{~V}$ in Reset, respectively (see Fig. 15).

In the considered RRAM cells the read signals has the same polarization of the Set operation (both pulses are applied on the BL), hence the read disturb could only be a problem on cells in HRS state because a very long sequence of read pulses could slowly re-create the conductive filament and change the state of the cells [31]. Thus, read disturb has been evaluated only on cells in HRS state: Fig. 15 shows the average HRS read current and its relative standard deviation measured during 100k read operations.

To evaluate the endurance properties of the cells, 10k Set/Reset cycles have been performed through an incremental program with verify procedure: such method leverages on the application of a sequence of pulses with increasing voltages, each step being followed by a current read operation that monitors the cell resistance [32]. When a cell reaches a predefined read current value after the pulse application, defined as verify target, the procedure is interrupted so that all cells are brought into a comparable electrical condition. In Set operation the BL pulse voltage was increased from $0.2 \mathrm{~V}$ to $3.5 \mathrm{~V}$ with steps of $0.1 \mathrm{~V}, T_{\text {pulse }}=10 \mu \mathrm{s}$, a WL voltage of $1.5 \mathrm{~V}$ and a readverify target $I_{\text {read }}=20 \mu \mathrm{A}$. In Reset operation the SL pulse voltage was increased from $0.2 \mathrm{~V}$ to $3.5 \mathrm{~V}$ with steps of $0.1 \mathrm{~V}$, $T_{\text {pulse }}=10 \mu \mathrm{s}$, a WL voltage of $2.5 \mathrm{~V}$ and a read-verify target $I_{\text {read }}=10 \mu \mathrm{A}$. The average values and standard deviations of the currents read after Reset (HRS) and Set (LRS) operations during the endurance test are shown in Fig. 17. A resume of the performance features of the proposed ATE with RRAM arrays is presented in Table II.

TABLE II

Measured ATE PeRformance EVALUATEd on RRAM FULL CHIP TESTS

\begin{tabular}{|l|c|}
\hline Test & Time $[\mathrm{h}]$ \\
\hline I-V Curves & 0.58 \\
Read disturb (1e5 cycles) & 1225 \\
Endurance $(1 \mathrm{e} 4$ cycles) & 6137 \\
\hline
\end{tabular}

\section{ATE BENCHMARKING}

Few commercial platforms [5], [9]-[11] have been presented for testing RRAM and MRAM devices, although those solutions are more suitable to test either single cell structures or
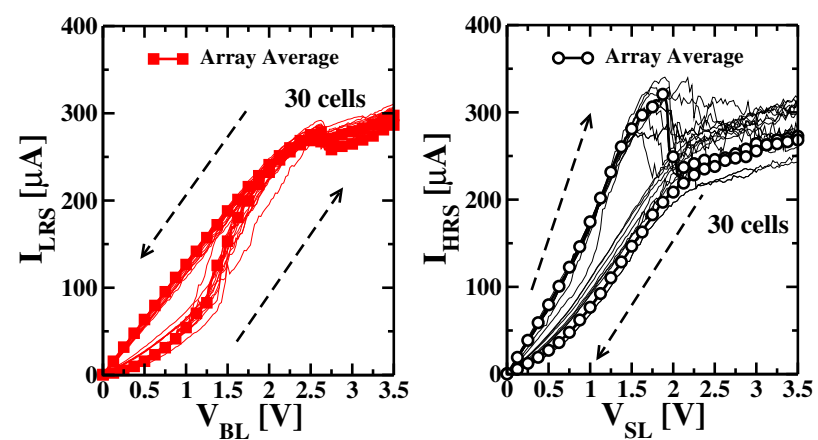

Fig. 15. SET (left) and RESET (right) switching kinetics performed on 30 cells in the RRAM array test structure [30].

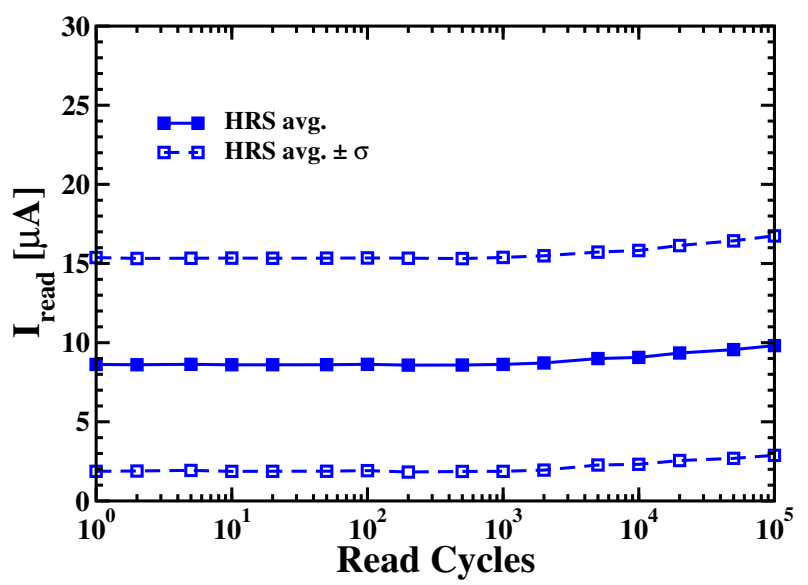

Fig. 16. Average read resistance and standard deviations for HRS state measured during a 100k cycles read disturb test on a full RRAM array.

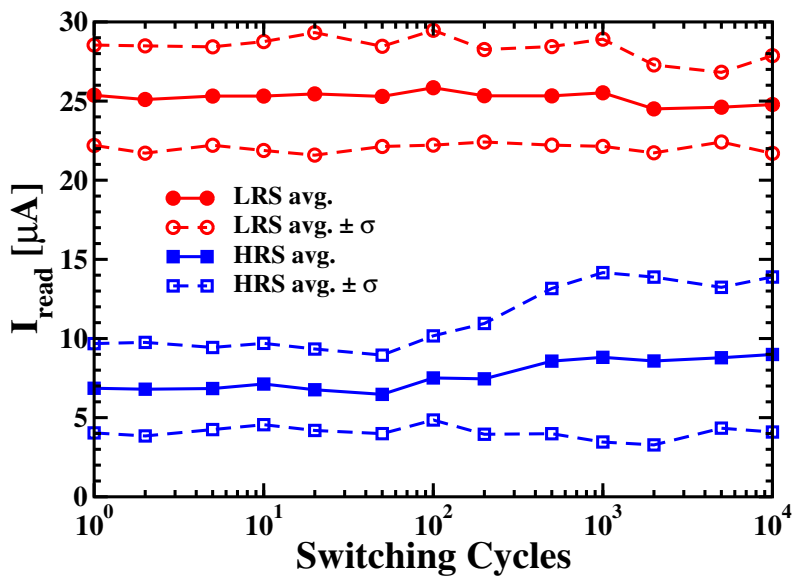

Fig. 17. Average read resistance and standard deviations measured on RRAM array at fixed switching cycles measured during an endurance test constituted by $10 \mathrm{k}$ switching cycles.

small test element groups. Moreover, the provided flexibility is often traded with the efficiency of test time. These test solutions require the interfacing with additional instrumentation and the usage of custom programming language that make difficult the fast evaluation of test patterns. In particular, the proposed ATE is proven to reach the performance of highend measurement systems such as the one demonstrated in [9] in terms of measurement resolution (i.e., hundreds of $\mu \mathrm{V}$ 

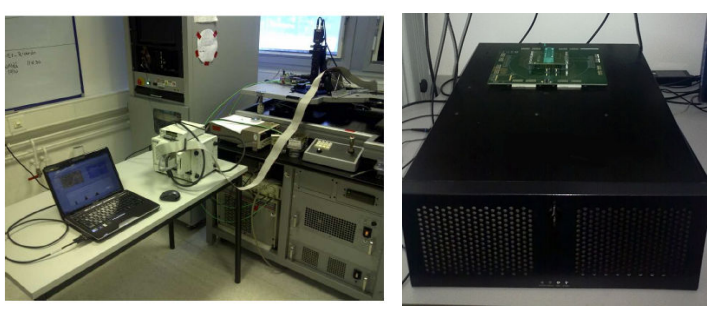

Fig. 18. Test platforms at a glance: the modular setup (left) and the proposed ATE (right).

using a $1.2 \mathrm{~V}$ full scale), number of parallel measurement channels (i.e., eight parallel measurement channels), and synchronization/triggering capabilities (i.e., delayed measurement triggered by external events). In this section, the developed ATE is benchmarked against a common modular setup that is used in characterization and qualification of TAS-MRAM arrays. Fig. 18 shows the two platforms at a glance.

The benchmark setup is composed by a PXI-based bench controlled by a PC via an ExpressCard plug. The control routines are written in MATLAB language. The PC controls all the cards inside the PXI chassis: the analog output card (NI-PXI 6723) [33] for the heating and switching voltages generation, the analog acquisition card for the sensing (NIPXI 6220) [34], and the GPIB card for data dispatching. The performance of this system are significantly lower than those offered by the proposed ATE: the analog output card has a sampling rate that limits the pulses generation for the heating and the switching voltages in the $\mu$ s-range, whereas the analog acquisition card has a timing resolution of 50ns that is four times higher than the performance achieved by the proposed ATE. Moreover, two points favor the developed ATE against this setup: the compactness of the system and the ease of programming. The former, because no external cables are foreseen in the proposed tester minimizing the risk of disturbs and measurement errors. The latter, because the intuitive GUI and the built-in post-analysis tools allow developing complex test flows in shorter time and with additional features like the run-time modification of the testing conditions during the test execution.

\section{CONCLUSIONS}

In this paper we have presented a dedicated automated test equipment to be used for the characterization of TAS-MRAM and RRAM array structures. Its internal hardware architecture has been developed using a modular approach that favors the integration between state-of-the-art PCI bus technology and cutting edge technology used for the development of high speed/high power arbitrary waveform generators. The instrument software is organized hierarchically so that lowlevel high-speed routines cohabit with graphical user interfaces that easily allows controlling all the hardware parameters. The performance of the tester has been proven in running both single cell tests and more complex routines, where a high parallelization degree was needed, with a relatively low execution time.

\section{REFERENCES}

[1] JESD22-A117C, "Electrically erasable programmable ROM (EEPROM) program/erase endurance and data retention stress test," 2011.

[2] P. Pellati and P. Olivo, "Automated test equipment for research on nonvolatile memories," IEEE Trans. on Instrumentation and Measurement, vol. 50, no. 5, pp. 1162-1166, 2001.

[3] D. Baderna, A. Cabrini, L. Gobbi, and G. Torelli, "A versatile and compact USB system for electrical and thermal characterization of non-volatile memories," in IEEE Instrumentation and Measurement Technology Conference (IMTC), Apr. 2006, pp. 1217-1220.

[4] Keithley, "Pulse I-V Characterization of Non-Volatile Memory Technologies."

[5] Integral Solutions International, "WLA-3000 STT-MRAM," 2012. [Online]. Available: http://www.us-isi.com/WLA-30001\%20STT-MRAM. htm

[6] C. Zambelli, A. Grossi, D. Walczyk, T. Bertaud, B. Tillack, T. Schroeder, V. Stikanov, P. Olivo, and C. Walczyk, "Statistical analysis of resistive switching characteristics in ReRAM test arrays," in IEEE Int. Conf. on Microelectronics Test Structures (ICMTS), Mar 2014, pp. 27-31.

[7] C. Zambelli, A. Grossi, P. Olivo, C. Walczyk, and C. Wenger, "RRAM Reliability/Performance Characterization through Array Architectures Investigations," in IEEE Computer Society Annual Symp. on VLSI (ISVLSI), July 2015, pp. 327-332.

[8] A. Grossi, C. Zambelli, P. Olivo, E. Miranda, V. Stikanov, T. Schroeder, C. Walczyk, and C. Wenger, "Relationship among current fluctuations during forming, cell-to-cell variability and reliability in RRAM arrays," in IEEE Int. Memory Workshop (IMW), May 2015, pp. 1-4.

[9] "Keysight 4082A Parametric Test System datasheet," 2014. [Online]. Available: http://literature.cdn.keysight.com/litweb/pdf/5989-6508EN. pdf

[10] "Keythley S530 Parametric Test Systems datasheet," 2014. [Online]. Available: http://www.keithley.com/data?asset=52643

[11] R. Robertazzi, J. Nowak, and J. Sun, "Analytical MRAM test," in IEEE Int. Test Conference (ITC), Oct 2014, pp. 1-10.

[12] A. Grossi, C. Zambelli, P. Olivo, P. Pellati, M. Ramponi, J. AlvarezHerault, and K. Mackay, "Automated characterization of TAS-MRAM test arrays," in IEEE Int. Conf. On Design Technology of Integrated Systems In Nanoscale Era (DTIS), April 2015, pp. 1-2.

[13] A. Grossi, C. Zambelli, P. Olivo, J. Alvarez-Herault, and K. Mackay, "Reliability and cell-to-cell variability of TAS-MRAM arrays under cycling conditions," in Non-Volatile Memory Technology Symposium (NVMTS), 2015, pp. 1-4.

[14] S. Chaudhuri, W. Zhao, J.-O. Klein, C. Chappert, and P. Mazoyer, "Design of TAS-MRAM prototype for NV embedded memory applications," in IEEE Int. Memory Workshop (IMW), May 2010, pp. 1-4.

[15] J. Azevedo, A. Virazel, A. Bosio, L. Dilillo, P. Girard, and S. Pravossoudovitch, "Test and reliability of magnetic random access memories," in GDR SOC-SIP, June 2011, pp. 1-2.

[16] J. Akerman, P. Brown, M. DeHerrera, M. Durlam, E. Fuchs, D. Gajewski, M. Griswold, J. Janesky, J. Nahas, and S. Tehrani, "Demonstrated reliability of 4-mb MRAM," IEEE Trans. on Device and Materials Reliability, vol. 4, no. 3, pp. 428-435, 2004.

[17] K. Lee and S. Kang, "Design Consideration of Magnetic Tunnel Junctions for Reliable High-Temperature Operation of STT-MRAM," IEEE Trans. on Magnetics, vol. 46, no. 6, pp. 1537-1540, 2010.

[18] E. Vatajelu, H. Aziza, and C. Zambelli, "Nonvolatile memories: Present and future challenges," in International Design Test Symposium (IDT), Dec 2014, pp. 61-66.

[19] J. Azevedo, A. Virazel, A. Bosio, L. Dilillo, P. Girard, A. Todri-Sanial, J. Alvarez-Hérault, and K. Mackay, "A Complete Resistive-Open Defect Analysis for Thermally Assisted Switching MRAMs," IEEE Trans. on Very Large Scale Integration (VLSI) Systems, vol. 22, no. 11, pp. 23262335, 2014.

[20] C.-L. Su, C.-W. Tsai, C.-W. Wu, C.-C. Hung, Y.-S. Chen, D.-Y. Wang, Y.-J. Lee, and M.-J. Kao, "Write Disturbance Modeling and Testing for MRAM," IEEE Trans. on Very Large Scale Integration (VLSI) Systems, vol. 16, no. 3, pp. 277-288, 2008.

[21] S. Senni, L. Torres, G. Sassatelli, A. Bukto, and B. Mussard, "Power efficient thermally assisted switching magnetic memory based memory systems," in Int. Symp. on Reconfigurable and Communication-Centric Systems-on-Chip (ReCoSoC), May 2014, pp. 1-6.

[22] S. F. Karg, G. I. Meijer, J. G. Bednorz, C. Rettner, A. G. Schrott, E. A. Joseph, C. H. Lam, M. Janousch, U. Staub, F. La Mattina, S. F. Alvarado, D. Widmer, R. Stutz, U. Drechsler, and D. Caimi, "Transition-metaloxide-based resistance-change memories," IBM Journal of Research and Development, vol. 52, no. 4.5, pp. 481-492, 2008. 
[23] D. Walczyk, T. Bertaud, M. Sowinska, M. Lukosius, M. A. Schubert, A. Fox, D. Wolansky, A. Scheit, M. Fraschke, G. Schoof, C. Wolf, R. Kraemer, B. Tillack, R. Korolevych, V. Stikanov, C. Wenger, T. Schroeder, and C. Walczyk, "Resistive switching behavior in TiN/HfO $2 /$ Ti/TiN devices," in Int. Semiconductor Conf. DresdenGrenoble (ISCDG), Sep. 2012, pp. 143-146.

[24] F. T. Chen, H. Y. Lee, Y. S. Chen, Y. Y. Hsu, L. J. Zhang, P. S. Chen, W. S. Chen, P. Y. Gu, W. H. Liu, S. M. Wang, C. H. Tsai, S. S Sheu, M. J. Tsai, and R. Huang, "Operation of oxygen vacancy-based RRAM's," Science China Information Sciences, vol. 54, no. 5, pp. 1073 1086, 2011.

[25] R. Sousa, I. Prejbeanu, D. Stanescu, B. Rodmacq, O. Redon, B. Dieny, J. Wang, and P. Freitas, "Tunneling hot spots and heating in magnetic tunnel junctions," Journal of Applied Physics, vol. 95, no. 11, pp. 6783 6785, 2004.

[26] I. Prejbeanu, M. Kerekes, R. Sousa, H. Sibuet, O. Redon, B. Dieny, and J.-P. Nozières, "Thermally assisted MRAM," Journal of Physics. Condensed Matter, vol. 19, no. 16, pp. 165 218-165 241, 2007.

[27] I. Prejbeanu, S. Bandiera, J. Alvarez-Hérault, R. Sousa, B. Dieny, and J. Nozières, "Thermally assisted MRAMs: ultimate scalability and logic functionalities," Journal of Physics D: Applied Physics, vol. 46, no. 7, p. 074002, 2013

[28] P. Lorenzi, R. Rao, and F. Irrera, "Forming kinetics in $\mathrm{HfO}_{2}$-based 438-443, 2013.

[29] A. Grossi, D. Walczyk, C. Zambelli, E. Miranda, P. Olivo, V. Stikanov, A. Feriani, J. Sune, G. Schoof, R. Kraemer, B. Tillack, A. Fox, T. Schroeder, C. Wenger, and C. Walczyk, "Impact of Intercell and Intracell Variability on Forming and Switching Parameters in RRAM Arrays," IEEE Trans. on Electron Devices, vol. 62, no. 8, pp. 2502 2509, 2015.

[30] C. Zambelli, A. Grossi, P. Olivo, D. Walczyk, J. Dabrowski, B. Tillack, T. Schroeder, R. Kraemer, V. Stikanov, and C. Walczyk, "Electrical characterization of read window in ReRAM arrays under different SET/RESET cycling conditions," in IEEE Int. Memory Workshop (IMW), May 2014, pp. 1-4.

[31] Y. Chen, H. Lee, P. Chen, P. Gu, C. Chen, W. Lin, W. Liu, Y. Hsu, S. Sheu, P.-C. Chiang, W. Chen, F. Chen, C. Lien, and M. Tsai, "Highly scalable hafnium oxide memory with improvements of resistive distribution and read disturb immunity," in IEEE Int. Electron Devices Meeting (IEDM), Dec 2009, pp. 1-4.

[32] A. Grossi, C. Zambelli, P. Olivo, E. Miranda, V. Stikanov, C. Walczyk, and $\mathrm{C}$. Wenger, "Electrical characterization and modeling of pulse-based forming techniques in RRAM arrays," Solid-State Electronics, vol. 115 Part A, pp. 17 - 25, 2016.

[33] "NI PXI-6723 datasheet," 2014. [Online]. Available: http://www.ni. com/datasheet/pdf/en/ds-157

[34] "NI PXI-6220 datasheet," 2014. [Online]. Available: http://www.ni. com/datasheet/pdf/en/ds-15 RRAM cells," IEEE Trans. on Electron Devices, vol. 60, no. 1, pp.

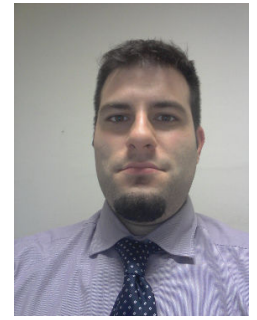

Cristian Zambelli (S'08-M'12) received the M.Sc. and Ph.D. degrees (with honors) in electronic engineering from Universitá degli Studi di Ferrara, Ferrara, Italy, in 2008 and 2012, respectively. Since 2015, he has been an Assistant Professor with Dipartimento di Ingegneria, Universitá degli Studi di Ferrara. His main research interests include the characterization, physics, and modeling of the reliability of nonvolatile memory devices, and algorithmic solutions for the reliability-performance tradeoff exploitation in solid-state drives.



Piero Olivo received the M.Sc. degree in electronic engineering and the Ph.D. degree from the University of Bologna, Bologna, Italy, in 1980 and 1987, respectively. Since 1994, he has been a Full Professor of Electronics with Dipartimento di Ingegneria, Universitá degli Studi di Ferrara, Ferrara, Italy. His scientific activity concerns the theoretical and experimental aspects of microelectronics, with an emphasis on the physics, reliability, and characterization of electron devices and nonvolatile memory devices.

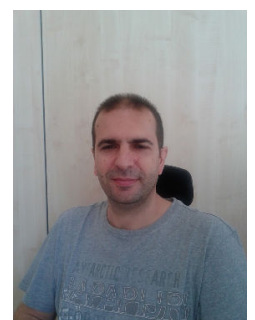

Paolo Pellati received the doctor degree in Electronic Engineering at the University of Ferrara, Ferrara, Italy in 1996. In 2000 he received his $\mathrm{PhD}$ degree in Electronic Engineering at the University of Modena and Reggio Emilia, Reggio Emilia, Italy. His $\mathrm{PhD}$ thesis concerned the study of characterization techniques for non-volatile memories and the development of a prototype of a test equipment dedicated to the characterization of nonvolatile memories at research level, named RIFLE. After his $\mathrm{PhD}$ he continued his activity related to the development of the RIFLE test equipment in co-operation with the University of Ferrara, where the RIFLE prototype was extensively used in research activities. In 2003 he co-founded Active Technologies. He is the company's President and he is responsible of all design activities.

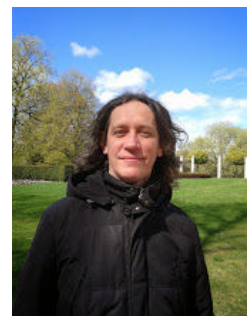

Michele Ramponi received the doctor degree in Electronic Engineering at the University of Ferrara, Ferrara, Italy in 2000. After an experience as system designer at Selcom SPA, in 2002 he joined the team developing the RIFLE test system at the University of Ferrara and in 2003 he co-founded Active Technologies. He is the company's Director of operations and he is responsible for the design of customized test solutions and equipment and for the design of high-speed board.

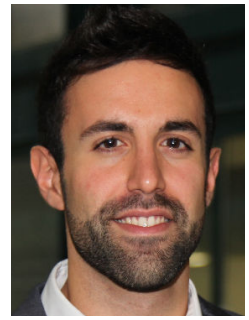

Alessandro Grossi received the M.Sc. degree in electronic and telecommunications engineering from Universitá degli Studi di Ferrara, Ferrara, Italy, in 2013 and is currently pursuing the Ph.D. degree in engineering science with Dipartimento di Ingegneria, Universitá degli Studi di Ferrara. His main research interests include the characterization, physics, and modeling of emerging non-volatile memory devices. 
Jérémy Alvarez-Hérault received the M.S. degree from the Grenoble Institute of Technology, Grenoble, France, in 2007, and the Ph.D. degree in physics from the University of Grenoble, Grenoble, in 2010. He joined Crocus Technology, Grenoble, in 2010, where he is currently a Lead Research and Development Engineer.

Ken Mackay received the Ph.D. degree in physics from the University of Cambridge, Cambridge, U.K. He was with Hitachi GST, San Jose, CA, USA

IBM, Armonk, NY, USA, and the National Center for Scientific Research, Paris, France. He is currently the Vice President of Technology Development with Crocus Technology, Grenoble, France. He has over 20 years of extensive research and development expertise in magnetoresistive materials. 